\title{
Universal Relationship Between Giant Magnetoresistance and Anisotropic Magnetoresistance in Spin Valve Multilayers
}

\author{
B. H. Miller $\mathbb{H}^{1}$, Branko P. Stojković, ${ }^{2}$ and E. D. Dahlberg ${ }^{1}$ \\ ${ }^{1}$ University of Minnesota, School of Physics and Astronomy, Minneapolis, MN 55455 \\ ${ }^{2}$ Center for Nonlinear Studies, Los Alamos National Laboratory, Los Alamos, NM 87545
}

\begin{abstract}
We measure the giant magnetoresistance (GMR) with the current both parallel and perpendicular to the direction of the magnetization in the ferromagnetic (FM) layers and thus probe the anisotropy of the effective mean free paths for the spin-up and spin-down electrons, seen in the anisotropic magnetoresistance. We find that the difference of the GMR in the two configurations, when expressed in terms of the sheet conductance, displays a nearly universal behavior as a function of GMR. On interpreting the results within the Boltzmann transport formalism we demonstrate the importance of bulk scattering for GMR.
\end{abstract}

The giant magnetoresistance (GMR) occurs in multilayers consisting of adjacent layers of FM and non-FM materials [1, 2, and is characterized by the large change in conductivity as the magnetic moments in the adjacent FM layers change from parallel to antiparallel. Experimentally, it has been determined that the amplitude of GMR depends strongly on the thickness of the constituent layers [3] and on the properties of the interfaces between them [4,5]. While there is a consensus that the spin-dependent scattering, present in FM materials [6 9], is responsible for the effect 10 14], it is still unclear whether the interfacial scattering or bulk scattering plays a more important role.

In this letter we report the results of a systematic study, aimed at sorting out the relative importance of the interfacial and bulk scattering processes. To accomplish this, we measure differences in two GMR configurations, one with the applied current parallel $\left(G M R_{\|}\right)$and the second with the current perpendicular $\left(G M R_{\perp}\right)$ to the magnetization of the sample. We find $G M R_{\perp}>G M R_{\|}$ for all 52 samples in this study. We attribute the difference in the two GMR values to the change in the difference in the mean free paths of spin-up and spin-down electrons in the two current/magnetization orientations. Since the current/magnetization orientation effect on the mean free paths of the spin-up and spin-down electrons, a phenomenon well known as the anisotropic magnetoresistance (AMR), is a bulk effect [15], we find that the GMR is also dominated by bulk spin-dependent scattering.

The films used in this study have been prepared by d.c. magnetron sputtering on $\mathrm{Si}(100) / \operatorname{SiN}_{x}(100 \mathrm{~nm})$ substrates, patterned for lift-off photo-lithography. They consist of two FM layers (made of Co and/or NiFe), separated by a non-magnetic ( $\mathrm{Cu}$ or $\mathrm{CuSn})$ spacer. A total of 52 samples, which can be divided into six series, have been measured (see Table 1). The top Co layer is allowed to partially oxidize in the air, creating a thin antiferromagnetic (AF) layer of $\mathrm{CoO}$, with a bulk Néel temperature $T_{N}$ of $\sim 290 \mathrm{~K}$. Magnetic moment measurements, obtained using a Quantum Design SQUID magnetometer, indicate that the top Co layer thickness is typically reduced by $t_{o x}=1-1.5 \mathrm{~nm}$ by oxidation [16]. When the samples are cooled to $T=4.2 K \ll T_{N}$ in a magnetic field of $2000 \mathrm{Oe}$, the AF layer provides a unidirectional exchange anisotropy, which pins the direction of the magnetization in the adjacent (top) FM layer, while in the bottom FM layer the magnetization is free to follow an applied field [17]. The orientation of the exchange anisotropy is easily altered by thermal cycling and resetting the applied field direction before cooling again.

We measure the GMR in both parallel and perpendicular configurations using the standard four-point probe technique with a well defined $(1 \mathrm{~mm} \times 10 \mathrm{~mm})$ conduction path [18]. We express the measured results in terms of sheet conductance, $G=\sigma t$, where $\sigma$ is the conductivity and $t$ is the film thickness. The four quantities necessary to describe the experiment are denoted by $G_{\| \uparrow}^{\uparrow \uparrow}, G_{\|}^{\uparrow \downarrow}, G_{\perp}^{\uparrow \uparrow}$, and $G_{\perp}^{\uparrow}$, where $\|$ and $\perp$ refer to the orientation of the magnetization relative to the current direction and $\uparrow$ and $\downarrow$ refer to the orientation of the magnetization in the ferromagnetic layers. Hence we define

$$
G M R_{\|}=G_{\|}^{\uparrow \uparrow}-G_{\|}^{\uparrow \downarrow}, \quad G M R_{\perp}=G_{\perp}^{\uparrow \uparrow}-G_{\perp}^{\uparrow \downarrow},
$$

and

$$
\Delta G M R=G M R_{\perp}-G M R_{\|} .
$$

Figure 11 shows the sheet conductance in a typical sample at $T=4.2 \mathrm{~K}$ for all states of interest: clearly, the four quantities are well defined, i.e., the energy of the exchange anisotropy of the pinned FM layer is high enough to provide a signal which is stable with increasing field strength. More importantly, the results show that the $G M R$ is larger in the perpendicular (higher conductance AMR) state. If one assumes that a Drude-like formalism can be applied, then this fact alone implies that the GMR is a probe of the difference of the mean free paths $\lambda$ of the spin-up $\left(\lambda^{\uparrow}\right)$ and spin-down $\left(\lambda^{\downarrow}\right)$ electrons $\left(\lambda^{\uparrow} \gg \lambda^{\downarrow}\right)$. In general, films with larger values of GMR are more sensitive to the change in the differences in mean free paths, caused by changing the magnetization from parallel to perpendicular relative to the current direction. 
In Figure 2, which is the central result of this paper, we plot $\triangle G M R$ as a function of $G M R_{\|}$(see Eq. (1)) in comparison with our calculated results (see below). The experimental data, plotted in terms of the sheet conductance, show striking universality up to rather large values of $G M R_{\|}$. Moreover the universality is not constrained only to different geometries of $\mathrm{Co} / \mathrm{Cu} / \mathrm{Co}$, the primary trilayer studied here, but is also applicable to different materials (see Table 1, series III and VI), as well as samples without a Ta seed layer (series IV). We emphasize that the relative change in GMR, defined as $G M R_{\perp,||}(\%)=1-G_{\perp,||}^{\uparrow \downarrow} / G_{\perp,||}^{\uparrow \uparrow}$ does not provide a universally behaved $\Delta G M R(\%) \equiv G M R_{\|}(\%)-G M R_{\perp}(\%)$, as seen in the inset of Figure 2. Hence, our result supports the view that the change in the sheet conductance, $G$, is the fundamental measure of GMR [19].

In order to eliminate possible systematic errors in the measurement technique we have performed a number of checks. For example, by replacing the $\mathrm{Cu}$ layer with a binary alloy $\mathrm{Cu}_{98} \mathrm{Sn}_{2}$ (series III in Table 1) whose bulk resistivity at $4.2 \mathrm{~K}$ is $\sim 6.2 \mu \Omega \mathrm{cm}$ which is somewhat larger than that in $\mathrm{Co}(\sim 5 \mu \Omega \mathrm{cm})$ and much larger than that of $\mathrm{Cu}$, we have verified that the behavior of $\triangle G M R$ as a function of $G M R$ is not due to current shunting by the copper, rather than the aforementioned change in the mean free paths. As seen in Figure 2, in this case the form of $\triangle G M R$ is virtually identical to that of films with a thin $\mathrm{Cu}$ spacer. Moreover, with the presumption that the interfaces in these polycrystalline samples are not atomically smooth, we can exclude internal reflection in the $\mathrm{Cu}[20$ as a possible explanation of the observed $\triangle G M R$. We have also examined whether the thermal cycling causes a shift in the base resistance, which could produce a non-vanishing value of $\triangle G M R$ observed in the experiment: we compared the value of $A M R=\rho_{\|}-\rho_{\perp}$, where $\rho_{\perp}$ and $\rho_{\|}$are obtained in two thermal cycles, to the AMR measured by rotating the magnetization in both FM layers in a large magnetic field $(10 \mathrm{kOe})$. In the latter case the resistivity was measured as a function of the angle $\theta$ between $\mathbf{j}$ and $\mathbf{M}$ (i.e., $A M R=\rho(\theta=0)-\rho(\theta=\pi / 2)$ ). This comparison yielded virtually no difference between the two values of the AMR and hence no systematic shifts in GMR due to thermal cycling.

For the analysis of our results, we use the semi-classical approach to GMR 10,14,21. This approach is similar to that of Rijks et al [22], although here we allow for spin dependent scattering at interfaces. Starting from the Boltzmann equation and neglecting deviations in Ohm's law, the displacement of the fermionic distribution function, $g(\mathbf{v}, z)=f-f_{o}$, is given by

$$
g_{i \pm}^{s}=\frac{e \tau_{i}^{s} E}{m_{i}^{s}}\left(\frac{\partial f_{0}}{\partial v_{x}}\right)\left[1-A_{i \pm}^{s}(\mathbf{v}) \exp \left(\frac{\mp z}{\tau_{i}^{s}\left|v_{z}\right|}\right)\right]
$$

where $e$ and $m$ are the electron charge and mass respectively, $v_{z}$ is the electron velocity perpendicular to the film plane, $\tau$ is the mean time between scattering events, $f$ is the steady state Fermi distribution, and $f_{o}$ is the equilibrium Fermi distribution. The functions $A_{i \pm}^{s}(\mathbf{v})$ are obtained from the following boundary conditions:

$$
\begin{aligned}
& g_{1+}^{s}\left(z=0, v_{z}\right)=p g_{1-}^{s}\left(z=0, v_{z}\right) \\
& g_{3-}^{s}\left(z=d, v_{z}\right)=q g_{3+}^{s}\left(z=d, v_{z}\right)
\end{aligned}
$$

and

$$
g_{(i \pm 1) \pm}^{s}\left(z_{i}, v_{z}\right)=T_{(i \rightarrow i \pm 1)}^{s} g_{i \pm}^{s}\left(z_{i}, v_{z}\right)
$$

where $\mathbf{v}$ is the quasiparticle velocity, $p$ and $q$ represent the fraction of electrons which are specularly scattered from the bottom and top surfaces respectively and $T_{(i \rightarrow i \pm 1)}^{s}$ are the transmission coefficients. The subscripts \pm indicate whether an electron is traveling in the positive or negative $z$ direction, and the superscript $s$ represents the spin state with respect to the magnetization in a FM layer.

From Eqs. (2)-(5) the sheet conductivity is straightforwardly obtained using

$$
G=\frac{J_{x} t}{E}=\frac{-2 e}{E}\left(\frac{m}{h}\right)^{3} \int_{0}^{t} d z \int_{-\infty}^{\infty} d^{3} v v_{x} g(\mathbf{v}, z)
$$

where $v_{x}, E_{x}$ and $J_{x}$ are the $x$-components of the velocity, applied electrical field and current respectively. It is easily verified that the "bulk" sheet conductance of the individual layers, $\sigma t_{i}$, where $t_{i}$ is the thickness of $i$ th layer, is subtracted out and does not contribute to the $G M R$ [16].

Equation (6) is most efficiently solved numerically. Clearly there are many parameters in the model, however, several experimental constraints can limit the parameter space considerably. For example, studies of the effect of surface scattering in polycrystalline thin films show that $p$ and $q$ are nearly zero [23]. In addition, our measured results show no substrate/seed layer dependence of $\triangle G M R$ vs $G M R$ and an explicit calculation shows that for $p, q<0.5$ our numerically obtained $\triangle G M R$ vs $G M R$ depends very little on $p$ and $q$. Hence we set $p=q=0$. In this limit one can obtain $G M R$ analytically and express the result in terms of exponential integrals, $E_{1}(x)$, where the argument $x$ depends only on ratios of thicknesses and mean free paths [16].

The coefficients $T_{(i \rightarrow i \pm 1)}^{s}$ represent the fraction of electrons specularly transmitted through an interface, with the subscript $i$ corresponding to the layer from which the electron was emitted. The remaining electrons are either diffusively transmitted or diffusively reflected, and in principle one should include the specular reflection coefficient $R$ into the calculation. However, in a polycrystalline sample, with presumably rough interfaces, electrons reflected at an interface will predominantly scatter 
diffusively and hence we set $R=0$. Although, strictly speaking, $T_{(i \rightarrow i \pm 1)}^{s}$ depend on both $i$ and $s$, here we assume that $T_{(i \rightarrow i \pm 1)}^{s}$ are the same at two interfaces; that is, we assume that the transmission coefficients do not depend on the incident layer $\left(T_{(1 \rightarrow 2)}^{s}=T_{(2 \rightarrow 1)}^{s}=T_{(2 \rightarrow 3)}^{s}=\right.$ $\left.T_{(3 \rightarrow 2)}^{s} \equiv T^{s}\right)$. Thus the eight transmission coefficients have been reduced to $T^{\uparrow}$ and $T^{\downarrow}$.

Within this model the observed $\triangle G M R$ is caused by the angular anisotropy of either the mean free paths or the transmission coefficients. Hence we extend the work of Ref. 21] and assume that in the magnetic layers (corresponding to indices $i=1,3) \lambda_{i}^{s}$ depend on the angle $\theta$ between $\mathbf{M}$ and $\mathbf{j}$ as in AMR, while in the non-magnetic interlayer the mean free path has neither a spin nor angular dependence, $\lambda_{2}(\theta)=\lambda_{2}=$ const. In principle, the transmission coefficients $T^{s}$ may exhibit anisotropy as well 24]; however, in the polycrystalline samples studied here this anisotropy is averaged to zero. Moreover, it is easy to verify, using the aforementioned analytical solution of Eq. (6), that the angular change in $T^{s}$, $\Delta T^{s}=T_{\|}^{s}-T_{\perp}^{s}$, yields $\Delta G M R \propto\left(\Delta T^{s} / T^{s}\right) G M R$ and no higher order terms, regardless of whether $G M R$ is due to the spin-dependent mean free paths or transmission coefficients, i.e., $\triangle G M R$ plotted against $G M R$ would yield a straight line. Hence, the experimentally observed curvature of $\triangle G M R$ vs GMR, seen in Fig. 2, is in clear contradiction with $\triangle G M R$ being due to the anisotropy of $T^{s}$, i.e., within this formalism the observed $\triangle G M R$ is due to the angular anisotropy of the conduction electron mean free paths in the ferromagnetic layers (the origin of the AMR). Indeed, with reasonable values of $\lambda_{\|}^{\uparrow}$ and $\lambda_{\perp}^{\uparrow}$, estimated from the the bulk resistivity anisotropy of Co at $T=4.2 \mathrm{~K}$, one can easily fit the data (see the solid line in Fig. 2).

Analytically, it is straightforward to show that, both for $\lambda^{\uparrow} \gg \lambda^{\downarrow}$ and/or $T^{\uparrow}>T^{\downarrow}$, one has $\triangle G M R \propto$ $\left(\Delta \lambda^{s} / \lambda^{s}\right) G M R$ plus higher order (in GMR) terms. Hence, the universal slope of $\triangle G M R$ at low $G M R$ indicates that the bulk magnetic scatterers (responsible for the AMR) dominate scattering in both magnetic materials used here (Co and $\mathrm{NiFe}$ ). In addition, for thin nonFM spacer our theoretical solution for $\triangle G M R$ does not depend on $\lambda_{2}$, in agreement with experiment. However, our experimental results show that $\triangle G M R$ as a function of $G M R$ is independent of both $\lambda^{s}$ and $T^{s}$. Both analytically and numerically one can show that this is possible only if $T^{\uparrow} \approx T^{\downarrow} 16$. Moreover, the sensitivity of the calculated result to the change in $T^{\uparrow}-T^{\downarrow}$ can be relatively large, and if the GMR is due to the spindependent transmission coefficients, then the experiment would likely show a non-monotonic behavior of $\triangle G M R$ vs $G M R$ even within the same series. Thus the observed universal behavior of $\triangle G M R$ implies that $T^{s}$ are only weakly spin dependent. From the amount of scatter in Fig. 2 we find that the difference between $T^{\uparrow}$ and $T^{\downarrow}$ is at most about $10 \%$.

The measured result for series II requires further explanation: in samples with $t_{C u}>4 \mathrm{~nm} \Delta G M R$ as a function of $G M R$ deviates from the otherwise universal behavior (see Fig. 2). Moreover, in the same series we find that $G M R$, expressed in terms of the sheet conductance (1), increases with increasing $\mathrm{Cu}$ thickness up to $t_{C u}=10 \mathrm{~nm}$, which is physically counter intuitive. However, the present model assumes that the mean free path in $\mathrm{Cu}$ does not depend on the $\mathrm{Cu}$ layer thickness. It is well known that $\lambda_{C u}$ depends on the grain size and that the grain sizes increase with increasing film thickness. Therefore it is reasonable to assume that $\lambda_{C u}$ increases with increasing $\mathrm{Cu}$ thickness. We have verified by explicit calculation that this indeed yields the experimentally observed result [16].

In conclusion, we have performed a systematic examination of the GMR effect in spin-valves in two configurations, with the applied current parallel and perpendicular to the magnetization direction. The obtained results are in agreement with the fact that the sheet conductance is the most fundamental measure of the magneto-transport in thin films and multilayers. We find that the difference of $G M R$ in the two configurations, $\Delta G M R$, is a universal function of $G M R$ and is due to the angular dependence of the electronic mean free path for spin-up and spindown electrons. We argue on physical grounds that the spin dependent transmission coefficients cannot yield the observed behavior. The universality of the behavior of $\triangle G M R$ vs $G M R$, both as a function of layer thickness and the mean free paths, demonstrates that bulk magnetic scatterers are primarily responsible for the GMR.

We wish to thank A. Balatsky for useful conversations. This work has been supported by the U.S. Department of Energy and by the AFOSR under grant no. AF/FA962092-J-0185.

* Current address: Seagate Technology, MS NRE 240, 1 Disc Drive, Minneapolis, MN 55435.

[1] G. Binasch, P. Gränberg, F. Saurenbach, and W. Zinn, Phys. Rev. B 39, 4828 (1989).

[2] C. Dupas, J. P. Renard, J. Seiden, E. Vélu, and D. Renard, J. Appl. Phys. 63 (8), 4300 (1988).

[3] B. Dieny, J. Magnetism and Mag. Mat. 136, 335, (1994).

[4] S. S. P. Parkin, Phys. Rev. Lett. 71, 1641 (1993).

[5] E. E. Fullerton, D. M. Kelly, J. Guimpel, and I. K. Schuller, Phys. Rev. Lett. 68, 859 (1992).

[6] N. Mott, Proc. R. Soc. 156, 368 (1936).

[7] N. Mott, Adv. Phys. 13, 325 (1964).

[8] I. A. Campbell and A. Fert, Ferromag. Mat. 3, 747 (1982).

[9] B. Gurney, V. S. Speriosu, J.-P. Nozieres, H. Lefakis, D. 
R. Wilhoit, and O. U. Need, Phys. Rev. Lett. 71, 4023 (1993).

[10] R. E. Camley and J. Barnas, Phys. Rev. Lett. 63, 664 (1989).

[11] P. M. Levy, S. Zhang, and A. Fert, Phys. Rev. Lett. 65, 1643 (1990).

[12] A. Barthélémy and A. Fert, Phys. Rev. B, 43, 13124 (1991).

[13] D. M. Edwards, R. B. Muniz, and J. Mathon, IEEE Trans. Mag. 27, 3548 (1991).

[14] J. Barnas, A. Fuss, R. E. Camley, P. Gränberg, and W. Zinn, Phys. Rev. B. 42, 8110 (1990).

[15] While the effective mean free path in a thin film exhibiting AMR may depend on its thickness, the anisotropy of $\rho$ remains the same and the AMR can be considered a bulk effect; see, e.g., T. R. McGuire and R. I. Potter, IEEE Trans. Mag., MAG-11, (4), 1018 (1975).

[16] Details of this study will be given elsewhere.
[17] Films of this type are frequently referred to as "spinvalves."

[18] For measurements without a well defined current path it is hard to establish the mean free path as a function of angle between the current and the magnetization; see, e.g., Dieny et al, J. Appl. Phys., 796370 (1996).

[19] B. Dieny, J.P. Nozieres, V.S. Speriosu, B.A. Gurney, and D.R. Wilhoit, Appl. Phys. Lett. 61, (17), 2111 (1992).

[20] W. H. Butler, X.-G. Zhang, D. M. C. Nicholson, T. C. Schulthess, and M. MacLaren, Phys. Rev. Lett. 76, 3216 (1996).

[21] B. Dieny, J. Phys.: Condens. Matter, 48009 (1992)

[22] Rijks et al, Phys. Rev. B51, 281 (1995).

[23] A. F. Mayadas, R. Feder, and R. Rosenberg, J. Vac. Sci. Technol. 6, 690, (1969); A. F. Mayadas, M. Shatzkes, Phys. Rev. B, 4, 1382 (1970).

[24] M. D. Stiles, J. Appl. Phys. 79 (8), 5805 (1996).

TABLE I. Thickness (in $\mathrm{nm}$ ) of the various layers in the samples. There are a total of 52 films, separated into six series. In Series V an attempt was made to keep the two Co layers of equal thickness after oxidation of the upper Co layer. Note that films with NiFe in Series VI contain a $0.5 \mathrm{~nm}$ layer of Co deposited on the exposed surface, necessary to provide the AF layer of $\mathrm{CoO}$.

\begin{tabular}{|c|c|c|c|c|c|c|c|}
\hline \hline Series & $\mathrm{Ta}$ & $\mathrm{Ni}_{81} \mathrm{Fe}_{19}$ & $\mathrm{Co}$ & $\mathrm{Cu}$ & $\mathrm{Cu}_{98} \mathrm{Sn}_{2}$ & $\mathrm{Co}$ & $\mathrm{Ni}_{81} \mathrm{Fe}_{19}$ \\
\hline I & 2.5 & 0 & $4-20$ & 2.5 & 0 & 4 & 0 \\
\hline II & 2.5 & 0 & 5 & $2-10$ & 0 & 4 & 0 \\
\hline III & 2.5 & 0 & $2.5-20$ & 0 & 2.3 & 4 & 0 \\
\hline IV & 0 & 0 & $2-20$ & 2.5 & 0 & 4 & 0 \\
\hline V & 0,5 & 0 & $2.7-10$ & $2.1-2.5$ & 0 & $4.5-11$ & 0 \\
\hline VI & 0 & $2.5,5$ & $0,0.5$ & 2.5 & 0 & $0,0.5$ & 0 \\
\hline \hline
\end{tabular}

FIG. 1. Typical sheet conductance vs applied field for the magnetization parallel (open circles) and perpendicular (filled circles) to the applied current. The data are obtained in Ta $2.5 \mathrm{~nm} / \mathrm{Co} 5 \mathrm{~nm} / \mathrm{Cu} 2.5 \mathrm{~nm} / \mathrm{Co}\left(4-t_{o x}\right) \mathrm{nm}$ film. For $H<0$ the magnetization of the two layers are parallel $(\uparrow \uparrow)$ and for $H>0$ and they are antiparallel $(\uparrow \downarrow)$. The GMR corresponds to $G^{\uparrow \uparrow}-G^{\uparrow \downarrow}$ with the applied current either parallel $(\|)$ or perpendicular $(\perp)$ to the sample magnetization. The data, together with SQUID magnetization measurements, indicate that the magnetic states in Co layers are well defined.

FIG. 2. $\Delta G M R=G M R_{\perp}-G M R_{\|}$versus $G M R_{\|}=G_{\| \uparrow}^{\uparrow \uparrow}-G_{\| \downarrow}^{\uparrow \downarrow}$, for the series depicted in Table 1 , where $G_{\perp(\|)}^{\uparrow \uparrow(\uparrow \downarrow)}$ is the sheet conductance measured in magnetically parallel (antiparallel) states and with the applied current perpendicular (parallel) to the sample magnetization; an estimated relative error is less than $1 \%$. The data display a general trend for all films, independent of material with only a deviation for the thickest films in series II. The solid line shows the calculated result, obtained with $\lambda_{\|}^{\uparrow}=30 \mathrm{~nm}, \lambda^{\downarrow}=3 \mathrm{~nm}, \lambda_{\perp}^{\uparrow}-\lambda_{\|}^{\uparrow}=1.2 \mathrm{~nm}$ and $T^{\uparrow}=T^{\downarrow}=0.5$ which corresponds to the GMR being due to bulk spin-dependent scattering. $\triangle G M R$ calculated using anisotropic transmission coefficients is always linear in $G M R$, as depicted by the dashed line, obtained with $\lambda_{(\|, \perp)}^{s}=30 \mathrm{~nm}, T_{\perp}^{\uparrow}=0.8, T_{\|}^{\uparrow}=0.81$ and $T^{\downarrow}=0.4$. In all calculations we have assumed that the mean free path in $\mathrm{Cu}$ is $\lambda_{C u}=50 \mathrm{~nm}$. The thickness of the various layers were chosen directly from Table 1 . Inset: $\Delta G M R$ vs the relative change in GMR (with GMR given in percent), for films depicted in Table 1, displays a non-universal behavior. 


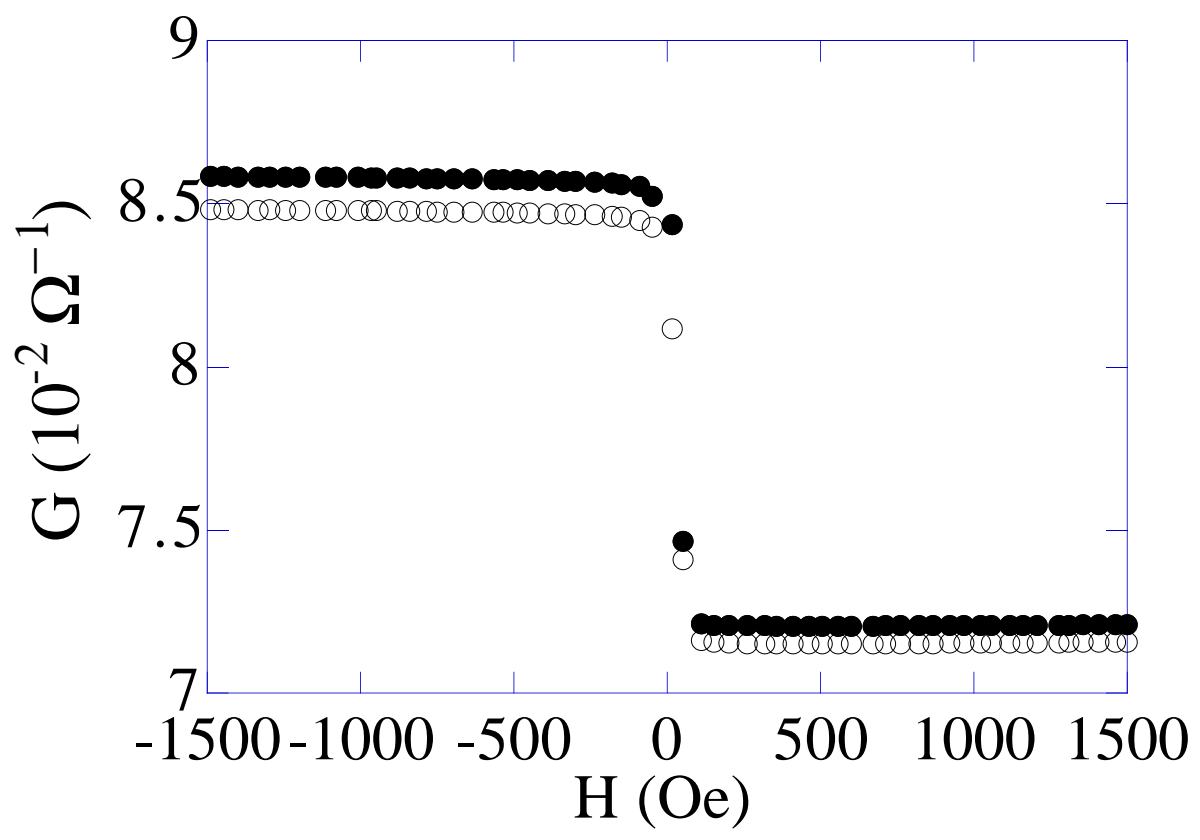





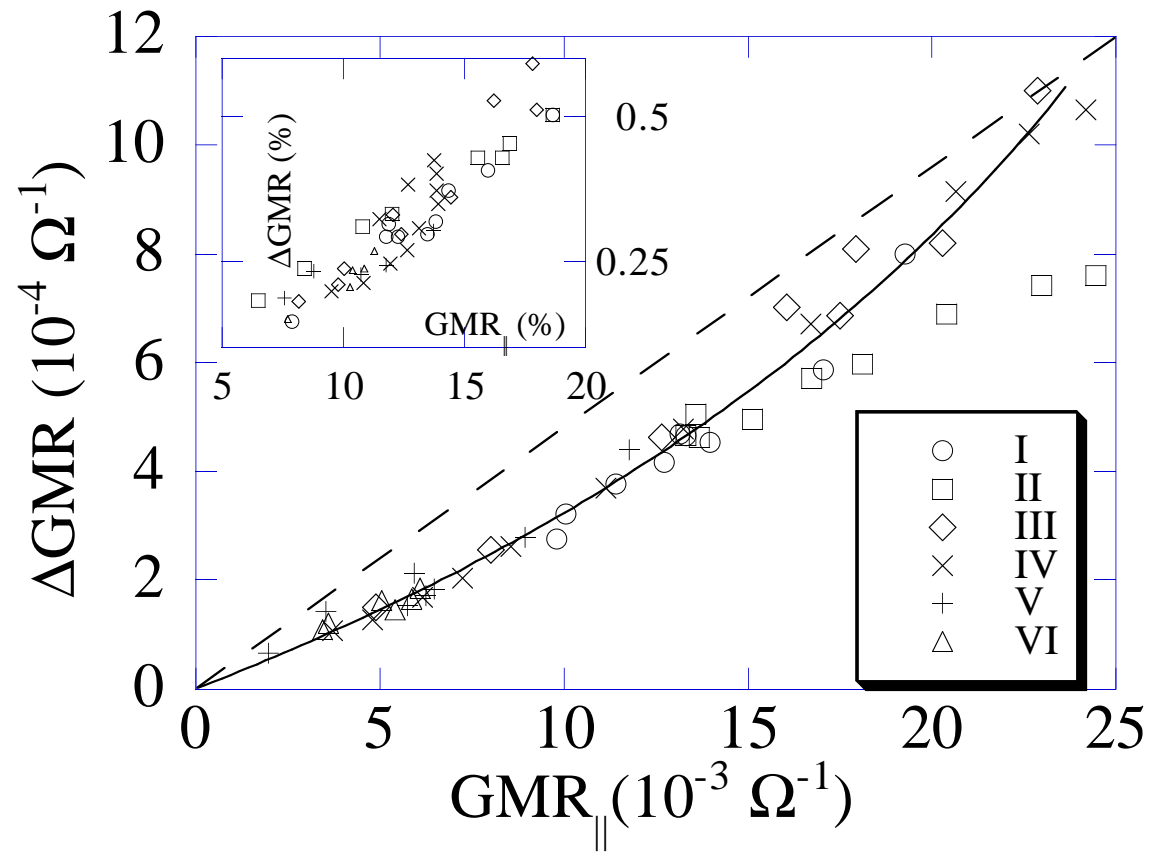


